\title{
Vehicle License Plate Detection Using Bounding Box Technique
}

\author{
Vanitha $\mathrm{N}^{\mathrm{a}, 1}$, Rene Robin C R ${ }^{\mathrm{b}}$, Doreen Hephzibah Miriam D ${ }^{\mathrm{c}}, \mathrm{NSankarGanesh}^{\mathrm{d}}$ \\ ${ }^{a}$ Research Scholar, Dept of CSE, Sri Sai Ram Engineering College, Chennai, India \\ ${ }^{b}$ Dept of CSE, Sri Sai Ram Engineering College, Chennai, India \\ ${ }^{c, d}$ Dept of CSE, Director of Computational Intelligence Research Foundation, India \\ Software Developer, Anandverce, Tamilnadu, India
}

\begin{abstract}
The use of vehicles in our daily lives is growing rapidly, and as more vehicles violate traffic laws, theft of vehicles, and a high number of incidents occur, crime rates rise linearly. Vehicle License Plate Detection (VLPD) is a image processing technology for recognizing the vehicle number plate. In a typical VLPD the image of the vehicle is first detected and the vehicle license plate region is captured. Then the filtering techniques are applied to the captured license plate region. In this study bounding box technique is applied for the segmentation and character recognition. It is observed that the created framework effectively detects and recognizes the vehicle number on different test pictures.
\end{abstract}

Keywords.Pre-Processing, Extraction, Segmentation, Recognition and VLPD.

\section{Introduction}

VLPD is required in automated traffic surveillance and vehicle monitoring due to the dramatic increase in the quantity of vehicles. However, with the advancement of digital cameras and the rise in computational capability over the last decade, it has sparked a lot of interest. The purpose of converting a vehicle number plate into a numerically readable character is simple. It can also be used to identify and deter a wide variety of illegal activities, as well as to maintain protection in highly restricted areas.Every vehicle has a unique identification number on its license plate. VLPD is extremely useful in detecting stolen cars, tracking illegal transportation, monitoring gates, speeding, and automatic parking. In order to recognize the vehicle in real time, VLPD must be fast. Despite the fact that there are many methods for identifying vehicle license plates, the performance of a VLPD system is determined by the image quality, which is deterioted by weather conditions, both static and dynamic. Mist, fog, and cloudiness are instances of static conditions, while downpour and snow are instances of dynamic conditions. Different challenges, like occlusion and shadow, often corrupt the VLPD recognition effectiveness.

${ }^{1}$ Vanitha N, Research Scholar, Dept of CSE, Sri Sai Ram Engineering College, Chennai, India.

Email: vanitha.sunder@gmail.com 


\section{Vlpd Challenges}

Real time VLPD is extremely difficult due to wide variety of plate design formats. Because of the differences in font size, it is clear that reading alpha numeric letters is difficult. Also, due to the screw fix, the digit ' 1 ' may be misread as ' 7, ' and the digit ' 7 ' may be misread as '9.'

\section{Related Works}

K. K. Kim et al., [1] deals with VLPD explicitly for Korean plates using Support Vector Machines and has a high normal character acknowledgment rate. Most optical character recognizers carried out in a 2D-plane just keep a high achievement rate only when the shooting distance of the image is less, as indicated by Ko et al., T. Naito et al., [2] [3].X. Pan et al., [4] proposed a multi-stage recognition scheme that combines statistical and structural recognition approaches, as well as distributing similar characters based on local structural features. Y. Huang et al., [5] used the gradient operator to find the likely number plate location. Parul Shah et al., [6] uses ANN for vehicle chassis number detection. N Vanitha et al., [7] segmented the tropical cyclone eye feature for the classification of cyclone based on the presence and absences of eye [8-13].

\section{Methodology}

There are four main stages to this methodology.
a. Pre-processing
b. Extracting
c. Segmentations
d. Recognition

a) Pre-processing: The pre-processing stage entails processing the raw image recorded with a digital camera before a particular part of the image is obtained.

b) Extraction: The raw image is converted to gray scale image, by applying various edge detections and threshold to image.

c) Segmentation: Consequently each character on the number plate are segmented using bounding box technique for detection.

d) Recognition: Finally, the procedure is used to equate the extracted image with the real characters in order to recognize the character. Figure 1 shows the block diagram 


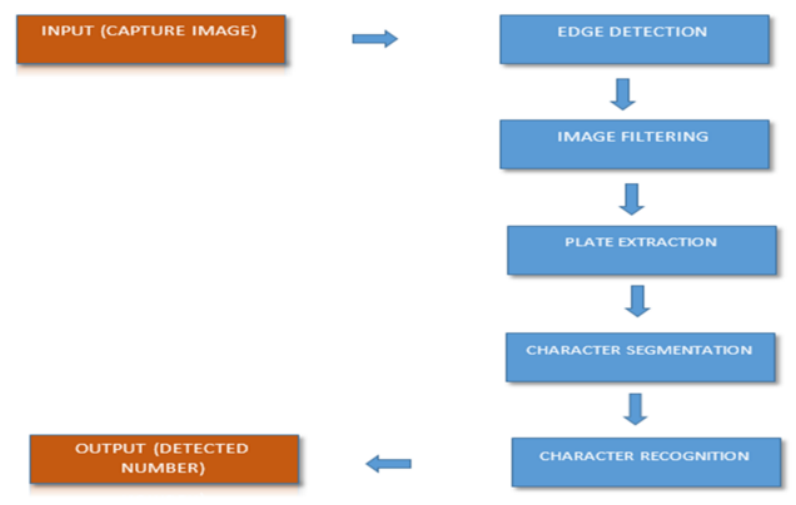

Figure 1.Block Diagram for VLPD

A) Collection of Image:In this analysis, 100 sample images is collected and tested for consistency. Figure 2, shows the input vehicle image with number plate.
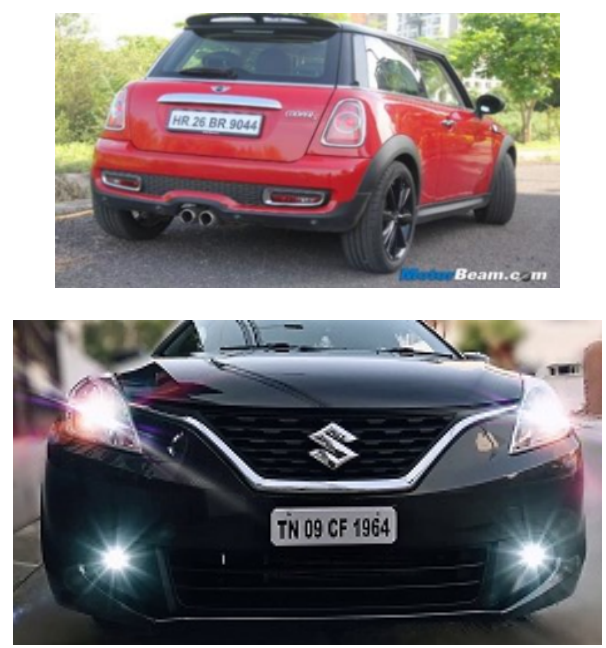

Figure 2.Collection of Vehicles with Number Plates

B) Pre-processing of Image: The picture, which was originally in RGB colour format, is converted to black and white. It will aid in the identification of the desired vehicle plate region and reduce the number of colours used in the picture. After that, the image converted with various edge detection techniques, and is followed by the process to extract the number plate very effectively and clearly.

C) Filtering of Image: The black and white images are subjected to the filtering process, after the colour image has been converted to binary. To filter and eliminate noise and distortion from the images, Mexican Hat filtering is chosen.

D) Extraction of Image: The desired features are then extracted from the filtered image using an image extraction of vehicle plate. The cropping process is be used in order to execute it. This approach would search for the digital image's black pixel.In this paper, we used the bounding box technique which can be used to place a strong topological prior on the solution, preventing it from shrinking too much and ensuring tightly bounded segmentation. 
E) Character Segmentation: Characters, letters, and numbers with a defined font make up the characters on vehicle licence plates. The main phases of character recognition include segmenting the vehicle license plate region, preprocessing, normalizing and using pattern matching for character recognition. Figure 7illustrates the character recognition of vehicle number plate region with OCR techniques.

\section{Experimental Results}

We have evaluated our method on the dataset of 100 images. Figure 3 shows the input image. Figure 4(a) - (d) illustrates applying filters to the input image.

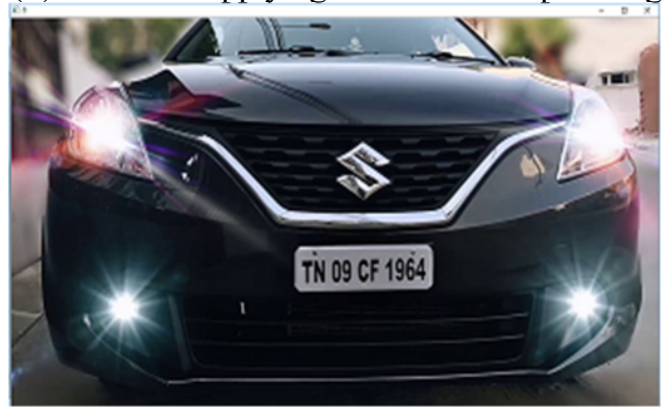

Figure 3.Input Image

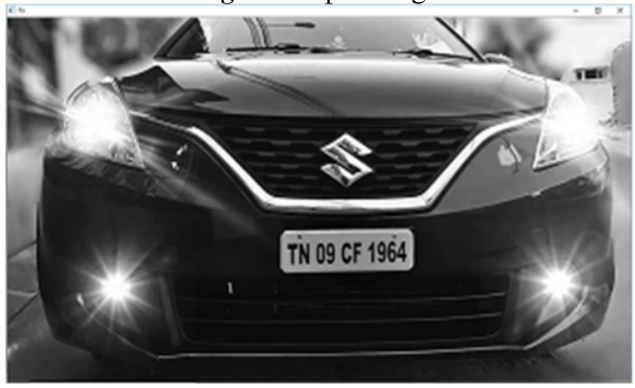

(a)

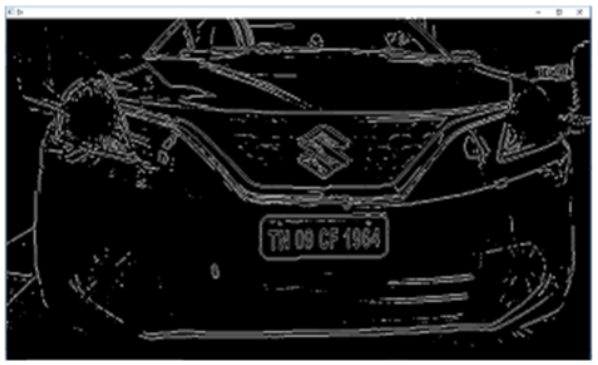

(b) 


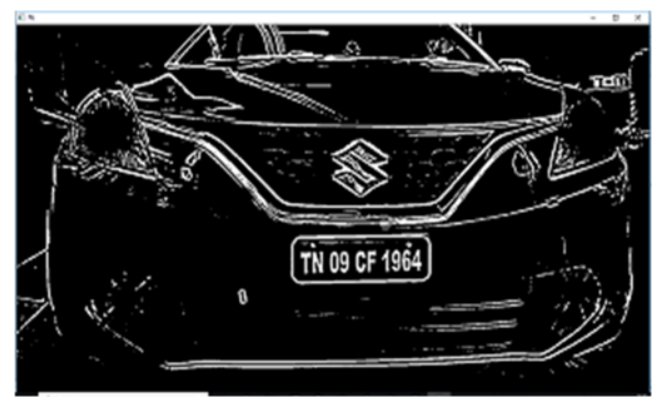

(c)

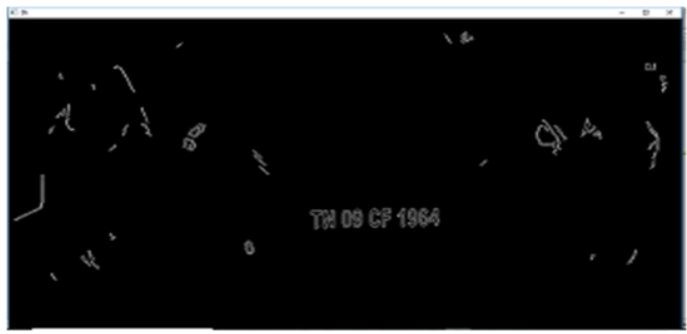

(d)

Figure 4.Applying Filters on image.

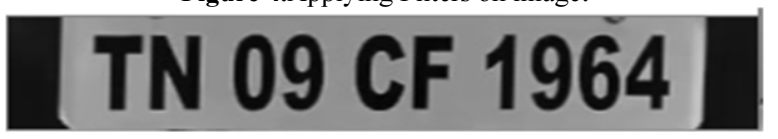

Figure 5.Extracting the vehicle license plate region using Bounding Box

\section{TN 09 CF 1964}

Figure 6.Applying the threshold in extracted plate region

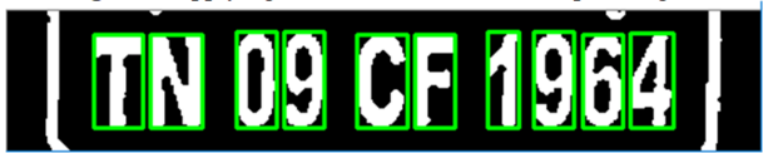

Figure 7.Segmentation using Bounding Box 


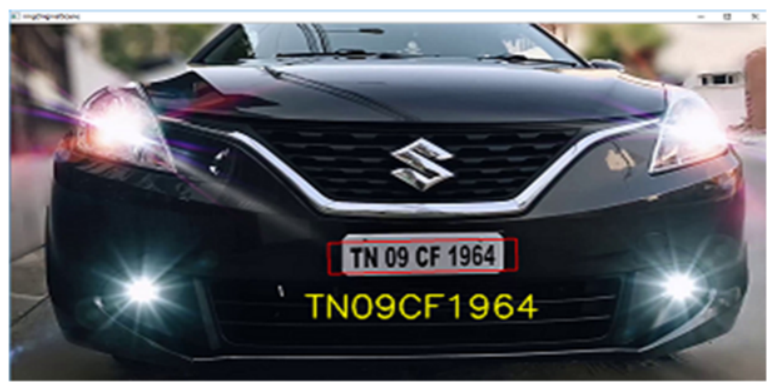

Figure 8.Recognizing the vehicle number

Figure 5 illustrates the vehicle license plate region extraction using Bounding Box technique. Figure 6 shows applying threshold to the extracted region. Figure 7 illustrates the character recognition. Finally figure 8 shows identifying the vehicle number of the input image. The digital image of the vehicle plate was successfully and efficiently processed, with the findings being shown and addressed. The study was run smoothly, and the key goal of developing an automatic vehicle plate detection system was met. Finally, the objective was achieved, and the Vehicle Number Plate was read, which is visible under the license plate region.

\section{Conclusion}

Thus we have investigated the existing frameworks that use license plate recognition systems, we have attempted to resolve system irregularities and achieved substantial results using the methods of various edge detection, threshold, segmentation, and character recognition. Our goal for potential enhancements is to upgrade the application so that it can provide better protection for the general public.Further work is to incorporate all conceivable complex cases of VLPD scenario. The most significant difference is that the state information is considered to be located at the top of the license plate. The majority of VLPDs concentrate on processing a single vehicle number plate, however in real-time, multiple vehicle number plates can be present when the images are being taken. A coarse-to-fine technique could be useful for segmenting multiple vehicle number plates.

\section{References}

[1] K. Kim, K. I. Kim, J. B. Kim and H. J. Kim, Learning-based approach for license plate recognition, in Proc. IEEE Signal Processing Society, Workshop, Neural Network Signal Process, Dec 2000, vol. 2, pp. 614-623.

[2] T. Naito, T. Tsukada, K. Yamada, K. Kozuka, and S. Yamamoto, Robust License-Plate Recognition Method for Passing Vehicle Under Outside Environment, IEEE Transactionson Vehicular Technology, Vol. 49, No.6, November 2000, pp. 2309-2319.

[3] M.A. Ko and Y.M. Kim, License Plate Surveillance System Using Weighted Template Matching, in Proc. of the 32nd Applied Imagery Pattern Recognition Workshop, October 2003, pp. 269-274.

[4] X. Pan, X. Ye and S. Zhang, A hybrid method for robust car plate character recognition . presented at the IEEE International Conference on Systems, Man and Cybernetics, 2004, pp. 4733-4737. 
[5] Y. Huang, S. Lai and W. Chuang, A Template-Based Model for License Plate Recognition , in The 2004 IEEE International Conference on Networking, Sensing \& Control, Taipei,2004,pp. 737-742.

[6] Parul Shah, Sunil Karamchandani, TaskeenNadkar, Nikita Gulechha, KaushikKoli, Ketan Lad, OCR-based ChassisNumber Recognition using Artificial Neural Networks , presented at the IEEE ICVES 2009, pp. 31-34.

[7] Vanitha, C R Rene Robin, Segmentation of Tropical Cyclone Eye Using Satellite Infrared Image ,International Journal of Recent Technology and Engineering (IJRTE), Volume-8, Issue-2S5, pp. 181-185, July 2019.

[8] Ramya,T.,Dr.Malathi,S.,ratheeksha,G.R. and Dr.V.D.Ambeth Kumar (2014). Personalized authentication procedure for restricted web service access in mobile phones. Applications of Digital Information and Web Technologies (ICADIWT), 2014, Page(s):69 - 74, Bangalore, India (ISBN:978-1-4799-2258-1)

[9] Ambeth Kumar.V.D (2018). Novel Wireless Sensing System For The Welfare Of Sewer Laborers. Healthcare Technology Letters (IET) Volume 5, Issue 4, p. $107-112$.

[10] Ambeth Kumar.V.D, G.Saranya , D.Elangovan, V.RahulChiranjeevi, V.D.Ashok Kumar.IOT Based Smart Museum Using Wearable Device.Lecture Notes in Networks and Systems, Vol.55, pp: 33-42, 2018

[11] S.Hema Kumar, J.UdayKiran, V.D.AKumar, G.Saranya, Ramalakshmi V.Effective Online Medical Appointment System.International Journal of Scientific \& Technology Research , Volume 8, Issue 09, September 2019, Pages 803 - 805.

[12] Ambeth Kumar.V.D, Dr.M.Ramakrishnan, V.D.Ashok Kumar and Dr.S.Malathi (2015) . Performance Improvement using an Automation System for Recognition of Multiple Parametric Features based on Human Footprint.for the International Journal of kuwait journal of science \& engineering, Vol 42, No 1 (2015), pp:109-132.

[13] Ambeth Kumar.V.D, V.D.Ashok Kumar, S.Malathi, K.Vengatesan, M.Ramakrishnan .Facial Recognition System For Suspect Identification Using A Surveillance Camera. Pattern Recognition and Image Analysis (Springer), Volume 28, Issue 3, pp 410-420, 2018. (DOI: 10.1134/S1054661818030136)

[14] V.D.Ambeth Kumar, "Precautionary measures for accidents due to mobile phone using IOT", Clinical eHealth, Volume 1, Issue 1, March 2018, Pages 30-35.

[15] B. Aravindh; V.D.Ambeth Kumar; G. Harish; V. Siddartth, " A novel graphical authentication system for secure banking systems”, IEEE (ICSTM), Pages: 177 - 183, 2-4 Aug. 2017, DOI: 10.1109/ICSTM.2017.8089147 